\title{
Fundamentos de la ventilación mecánica en el síndrome de distrés respiratorio agudo
}

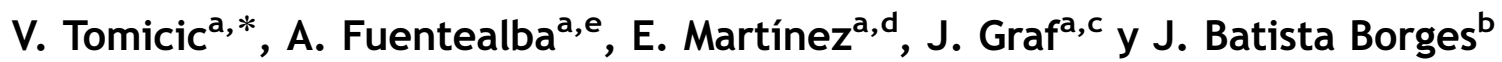 \\ a Unidad de Cuidados Intensivos, Clínica Alemana de Santiago, Unidad de Paciente Crítico, Hospital Padre Hurtado, Facultad \\ de Medicina Clínica Alemana, Universidad del Desarrollo, Santiago, Chile \\ ${ }^{\mathrm{b}}$ Department of Medical Sciences, Clinical Physiology University Hospital, Uppsala, Suecia, Departamento de Enfermedades \\ Respiratorias, Facultad de Medicina, Universidad de Sao Paulo, Sao Paulo, Brasil \\ ${ }^{c}$ Pulmonary Research Fellow, Regions Hospital, University of Minnesota, Minniapolis, Saint Paul, Minnesota, Estados Unidos \\ dMedicina Intensiva del Adulto, Facultad de Medicina Clínica Alemana, Universidad del Desarrollo, Santiago, Chile \\ e Facultad de Medicina Clínica Alemana, Universidad del Desarrollo, Santiago, Chile
}

Recibido el 24 de junio de 2009; aceptado el 21 de octubre de 2009

Disponible en Internet el 25 de enero de 2010

\author{
PALABRAS CLAVE \\ Síndrome de distrés \\ respiratorio agudo; \\ Ventilación mecánica; \\ Tomografía axial \\ computarizada; \\ Lesión asociada a \\ ventilador; \\ Colapso alveolar
}

\begin{abstract}
Resumen
El síndrome de distrés respiratorio agudo (SDRA) corresponde al daño de la barrera endotelioepitelial pulmonar inducida por inflamación, cuyo resultado condiciona aumento de la permeabilidad vascular y disfunción del agente tensioactivo y produce grados variables de colapso y relleno alveolar. Actualmente, el tratamiento consiste en ventilación mecánica. El desafío actual apunta a determinar qué estrategias ventilatorias son capaces de minimizar la lesión producida por el ventilador (VILI, ventilator-induced lung injury) y a procurar un intercambio gaseoso razonable.

La evidencia muestra que debería ventilarse a los pacientes con volumen tidal entre 6$8 \mathrm{ml} / \mathrm{kg}$, peso ideal con presión "meseta" $<30 \mathrm{cmH}_{2} \mathrm{O}$. El uso de altos niveles de presión positiva espiratoria final (PEEP, positive end expiratory pressure) no ha demostrado reducir la mortalidad; sin embargo, ha mejorado metas secundarias importantes. La racionalidad en el uso de niveles elevados de PEEP se fundamenta en su capacidad de reducir el colapsoreapertura cíclico de la vía aérea, probablemente el mayor culpable del desarrollo de VILI. La tomografía computarizada de tórax ha contribuido en la comprensión anatomicofuncional de los patrones de distribución del SDRA. La tomografía de impedancia eléctrica, aunque aún está en desarrollo, es una técnica libre de radiación capaz de monitorizar dinámicamente la ventilación al lado del enfermo.
\end{abstract}

(c) 2009 Elsevier España, S.L. y SEMICYUC. Todos los derechos reservados.

\footnotetext{
*Autor para correspondencia.

Correo electrónico: vtomicic@gmail.com (V. Tomicic).
} 


\section{KEYWORDS}

Acute respiratory

distress syndrome;

Mechanical

ventilation;

Computed

tomography scan;

Ventilator-induced

lung injury;

Lung collapse

The basics on mechanical ventilation support in acute respiratory distress syndrome

\begin{abstract}
Acute Respiratory Distress Syndrome (ARDS) is understood as an inflammation-induced disruption of the alveolar endothelial-epithelial barrier that results in increased permeability and surfactant dysfunction followed by alveolar flooding and collapse. ARDS management relies on mechanical ventilation. The current challenge is to determine the optimal ventilatory strategies that minimize ventilator-induced lung injury (VILI) while providing a reasonable gas exchange.

The data support that a tidal volume between $6-8 \mathrm{ml} / \mathrm{kg}$ of predicted body weight providing a plateau pressure $<30 \mathrm{cmH}_{2} \mathrm{O}$ should be used. High positive end expiratory pressure (PEEP) has not reduced mortality, nevertheless secondary endpoints are improved. The rationale used for high PEEP argues that it prevents cyclic opening and closing of airspaces, probably the major culprit of development of VILI.

Chest computed tomography has contributed to our understanding of anatomic-functional distribution patterns in ARDS. Electric impedance tomography is a technique that is radiation-free, but still under development, that allows dynamic monitoring of ventilation distribution at bedside.
\end{abstract}

(c) 2009 Elsevier España, S.L. and SEMICYUC. All rights reserved.

“...el científico tiene como misión encontrar la explicación más simple posible para la causa que está siendo observada..."

William de Ockham—1300 d.C.

\section{Introducción}

Cuarenta años de cuantiosa investigación sobre lesión pulmonar aguda (LPA) y síndrome de distrés respiratorio agudo (SDRA) ${ }^{1}$ no han sido suficientes para mejorar sustancialmente los resultados ${ }^{2-4}$. Es más, aún existe polémica tanto sobre conceptos fisiopatológicos determinantes como con relación a las estrategias terapéuticas actualmente disponibles ${ }^{5,6}$.

Esta entidad es una causa frecuente e importante de morbimortalidad ${ }^{4,7}$, y aquellos pacientes que sobreviven a menudo persisten con daño pulmonar y experimentan una disminución significativa en su calidad de vida. La mortalidad oscila entre un 31 y un $74 \%$, dispersión que se ha atribuido a las diferentes definiciones usadas para seleccionar los pacientes, a los datos provenientes de estudios observacionales y como consecuencia de la menor mortalidad exhibida por los estudios aleatorizados, en los que el cuidado que reciben los pacientes es más estrecho. En la Unión Europea la prevalencia de LPA/SDRA supera los 300.000 casos por año y la mortalidad bordea el $40 \%{ }^{8}$.

La lesión pulmonar puede gatillarse tanto por daño directo (neumonía, aspiración) como indirecto (sepsis, pancreatitis) sobre el parénquima pulmonar, y causar alteraciones de la barrera endotelial y cambios en el epitelio alveolar. Ambos fenómenos determinan una alteración profunda de la micromecánica alveolar producto del colapso gravitacional que impone el edema intersticial y de la inestabilidad alveolar secundaria a la depleción del agente tensioactivo, depósito de proteínas y presencia de detritus en la superficie alveolar ${ }^{9-11}$.

Se ha estudiado la permeabilidad vascular pulmonar cuantificando la fuga y acumulación consecuente de sustancias radioactivas en el intersticio pulmonar. Aunque en los pacientes con SDRA se ha observado un gradiente ventrodorsal marcado, el incremento de la permeabilidad se desarrolla a través de todo el pulmón, es decir, la agresión funcional determinada a través del incremento del agua pulmonar extravascular está presente también en regiones pulmonares libres de daño estructural ${ }^{12}$.

Se ha establecido que la ventilación mecánica (VM) por sí misma puede dañar el pulmón, concepto conocido como lesión producida por el ventilador (VILI, ventilador-induced lung injury) ${ }^{13-15}$. Esta susceptibilidad depende de la distribución heterogénea del deterioro pulmonar, lo que promueve la insuflación anisotrópica, la apertura alveolar fuera de fase y el colapso espiratorio final; éstos promueven el daño pulmonar, desencadenan una respuesta inflamatoria a distancia y determinan, así, el desarrollo de disfunción orgánica múltiple (DOM) ${ }^{16,17}$.

Por lo mismo, gran parte de la investigación en SDRA se ha dirigido a identificar una estrategia ventilatoria que respete la capacidad de aireación del pulmón enfermo, a la exploración del potencial para reclutamiento (PPR) y al ajuste de la presión positiva espiratoria final (PEEP, positive end expiratory pressure), sin otra intención que la de interrumpir el desarrollo de VILI y DOM.

Estudios clínicos han demostrado que la mortalidad asociada con LPA/SDRA puede reducirse con estrategias de ventilación que eviten el estiramiento excesivo del tejido pulmonar. Actualmente contamos con diversas estrategias ventilatorias; no obstante, la sugerida por la ARDS Network (ARDSnet) y la aproximación con pulmón abierto (open lung approach) son las más ampliamente difundidas ${ }^{2,3}$.

El objetivo de esta revisión es analizar los mecanismos causantes de la reducción del volumen de aireación y describir los fundamentos de las principales estrategias ventilatorias propuestas en los últimos años.

\section{Lesión pulmonar producida por el ventilador}

Aunque existen diversas causas potenciales de VILI, ésta se ha vinculado principalmente con 2 fenómenos: el primero, ocurre al final de la inspiración y se asocia con el uso de 
volumen tidal $\left(\mathrm{V}_{\mathrm{T}}\right)$ o presión inspiratoria elevados, que promueven sobredistensión alveolar, proceso relacionado con riesgo de rotura de la vía aérea y paredes alveolares en las regiones no dependientes (R-ND) del pulmón ${ }^{18,19}$. El segundo, se desarrolla esencialmente en las regiones dependientes (R-D), que están expuestas a estrés significativo cuando la vía aérea y los alvéolos se abren en inspiración y colapsan en espiración (tidal recruitment). Éste se asocia principalmente con el uso de PEEP insuficiente para evitar el colapso-reapertura alveolar cíclico ${ }^{20,21}$. Ambos fenómenos pueden superponerse, ya que la PEEP innecesariamente elevada puede condicionar sobredistensión, y la reducción del $\mathrm{V}_{\mathrm{T}}$ podría limitar el colapsoreapertura alveolar cíclico. En este sentido, es posible que el beneficio observado con el uso de $V_{\mathrm{T}}$ bajo en el estudio ARDSnet $^{3}$ no obedezca exclusivamente al control de la sobredistensión.

Hay falta de consenso acerca de la contribución precisa, cinética y rol primario de cada uno de estos mecanismos. La hipótesis de que el colapso del pulmón es perjudicial durante LPA/SDRA es aún discutible ${ }^{22}$. Sin embargo, estudios experimentales demuestran que el colapso de los espacios aéreos y el reclutamiento cíclico son elementos cruciales en el desarrollo de $\mathrm{VILI}^{20,21}$. Para empeorar las cosas, alrededor de un $10-20 \%$ de los pacientes que reciben VM sin diagnóstico inicial de LPA/SDRA están expuestos a VILI ${ }^{20-22}$.

En este contexto, permitir que la vía aérea y los alvéolos permanezcan colapsados u ocupados con líquido, obstaculizando la ventilación de extensas regiones pulmonares ("atelectasia permisiva”), no ha demostrado ser superior a la aplicación de maniobras de reclutamiento pulmonar (MRP). Más aún, las zonas de "atelectasia permisiva" son incapaces de eliminar secreciones, cuyo confinamiento es causa conocida de inflamación ${ }^{23-25}$.

\section{Teorías causantes de la reducción de la capacidad de aireación}

Los mecanismos más aceptados que determinan una limitación del volumen pulmonar para recibir el $\mathrm{V}_{\mathrm{T}}$ insuflado (pulmón pequeño o baby lung) son el edema intersticial y la inundación alveolar ${ }^{26,11}$. El primero se caracteriza por una disminución de la capacidad residual funcional (CRF) secundaria a pérdida de gas, condicionada por los efectos que ejerce el gradiente hidrostático (presión sobreimpuesta) sobre el tejido pulmonar y que conduce a colapso alveolar. Conocida con el nombre de "pulmón de esponja" (sponge lung), esta teoría se caracteriza por la incorporación de nuevas unidades alveolares durante una insuflación, efecto que se manifiesta con restablecimiento de la CRF y presencia de "reclutamiento" alveolar. En el segundo mecanismo, la CRF no se modifica con la aplicación de PEEP, debido a que existe ocupación de unidades alveolares por parte de proteínas y detritus, factor que previene el colapso. Esta teoría se conoce como "inundación alveolar" (alveolar flooding). Esta ocupación limita el reclutamiento, de tal manera que durante una insuflación el volumen se distribuye hacia las zonas normalmente aireadas; por este motivo, el aumento del volumen pulmonar ocurre principalmente a expensas de un incremento en el radio alveolar de las unidades previamente abiertas, lo que favorece la "sobredistensión"11,19,26,27 (fig. 1).

En consecuencia, ambos mecanismos muestran un factor causante común en el desarrollo de VILI: el "estrés" mecánico global o regional aplicado sobre un pulmón con escasa capacidad de aireación, donde las estructuras susceptibles son el fibroesqueleto pulmonar (FP), la microvasculatura, la vía aérea terminal y los delicados tejidos yuxtaalveolares.

El FP está constituido por fibras extensibles (elastina) e inextensibles (colágeno) en cuya vecindad se encuentran ancladas, vía integrinas, las células endoteliales y epiteliales (neumocitos I y II), donde el límite de la distorsión celular (stop length) viene determinado por las fibras colágenas. El FP responde con un incremento de su tensión de igual magnitud y en sentido opuesto a la presión aplicada por el ventilador; sin embargo, la verdadera causante de la distensión no es la presión de la vía aérea, sino la presión transpulmonar $\left(\mathrm{P}_{\mathrm{TP}}\right)$, que corresponde a la diferencia entre las presiones alveolar $\left(\mathrm{P}_{\mathrm{ALV}}\right)$ y pleural $\left(\mathrm{P}_{\mathrm{PL}}\right)$. Si superamos la $\mathrm{P}_{\mathrm{TP}}$ fisiológica, se produce el "estrés" o tensión mecáni$\mathrm{Ca}^{28,29}$ (fig. 2).

El pulmón normal duplica su volumen de reposo al alcanzar el $80 \%$ de la capacidad pulmonar total y este nivel se considera como límite superior del despliegue fisiológico del FP. Superar este límite condicionaría lo que conocemos como strain y su magnitud puede expresarse mediante el coeficiente entre el $\mathrm{V}_{\mathrm{T}}$ insuflado y el volumen pulmonar espiratorio final (EELV, end expiratory lung volume), es decir, $\mathrm{V}_{\mathrm{T}}$ dividido por el tamaño del baby lung ${ }^{26,27}$.

Para comprender mejor el origen de la lesión mecánica debemos conocer el concepto de elastance pulmonar específica (EPE), que considera tanto tensión como elongación y corresponde a la presión en la que el baby lung duplica su tamaño, es decir, cuando el coeficiente $\mathrm{V}_{\mathrm{T}} /$ baby lung es igual a uno $E P E=P_{T P}$ un valor superior a uno indica presencia de stress y strain. Gattinoni mostró que la EPE en el SDRA es similar a la de un pulmón normal (pulmón pequeño y no rígido) y corresponde aproximadamente a una $\mathrm{P}_{\mathrm{TP}}$ de $12,5 \mathrm{cmH}_{2} \mathrm{O}$, umbral que podría considerarse seguro durante la aplicación de VM. Cabe destacar que esta fórmula, al no considerar la presencia de reclutamiento, puede sobreestimar el strain $^{28}$.

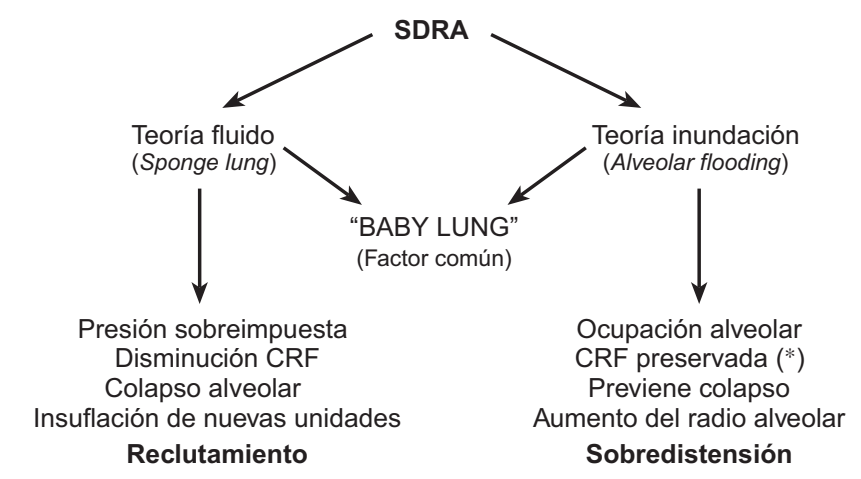

Figura 1 Mecanismos causantes de la reducción en la aireación pulmonar en el síndrome de distrés respiratorio agudo (SDRA) $\left.{ }^{*}\right)$ : considerada como el contenido de aire, tejido, edema y sangre. CRF: capacidad residual funcional. 

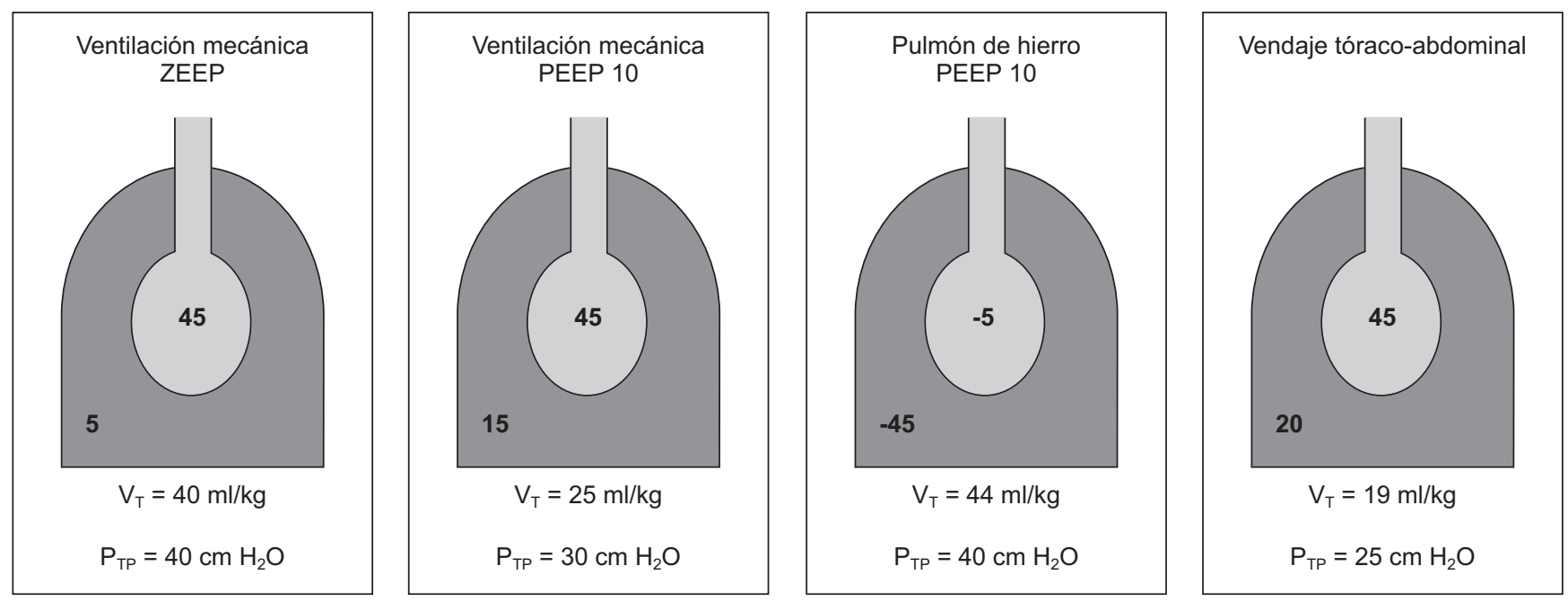

Figura 2 Interpretación de la presión transpulmonar en un modelo animal con diferentes condiciones ventilatorias. Descripción del trauma transpulmonar basados en una interpretación de los resultados obtenidos por Dreyfuss et al ${ }^{14}$. PEEP: positive end expiratory pressure; ZEEP: zero end expiratory pressure.

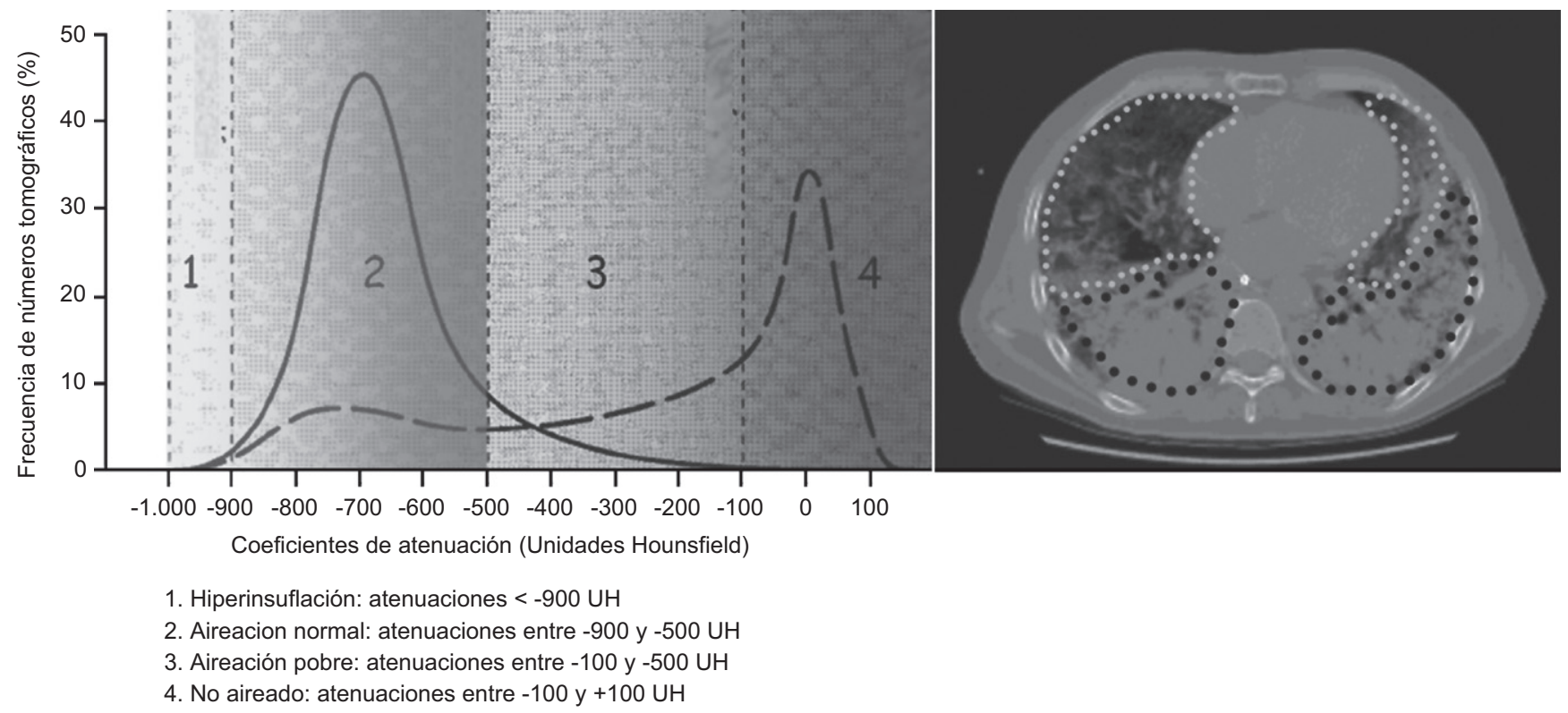

Figura 3 Distribución de la aireación pulmonar con tomografía computarizada en la lesión pulmonar aguda y el síndrome de distrés respiratorio agudo.

\section{Distribución regional del pulmón con síndrome de distrés respiratorio agudo}

\section{Tomografía computarizada de tórax}

La magnitud de la VILI se vincula con la distribución anatomofuncional heterogénea del tejido pulmonar. Estudios con tomografía computarizada (TC) de tórax han descrito 4 regiones: a) normalmente aireada, localizada principalmente en posición anterosuperior; b) pobremente aireada, caracterizada por opacidades tipo vidrio esmerilado en posición media; c) no aireada $o$ consolidada, distribuida en posición posterobasal, y d) hiperinsuflada (<-900 unidades Hounsfield) extrapolada a partir de la descripción del enfisema pulmonar ${ }^{10,29,30}$ (fig. 3).

Dadas las características lineales de la emisión de los rayos $\mathrm{X}$ y del algoritmo de reconstrucción (back projection) utilizado por la TC, la imagen obtenida en un mismo nivel tomográfico no representa necesariamente la misma zona de tejido pulmonar estudiada en diferentes condiciones ventilatorias (modificaciones del $\mathrm{V}_{\mathrm{T}}$ y PEEP). Además, los cambios en los coeficientes de atenuación (unidades Hounsfield) representan la composición de la densidad en cada voxel (unidad volumétrica mínima), y por tanto, la TC no puede distinguir entre colapso e inundación alveolar ${ }^{29}$ (fig. 4). 


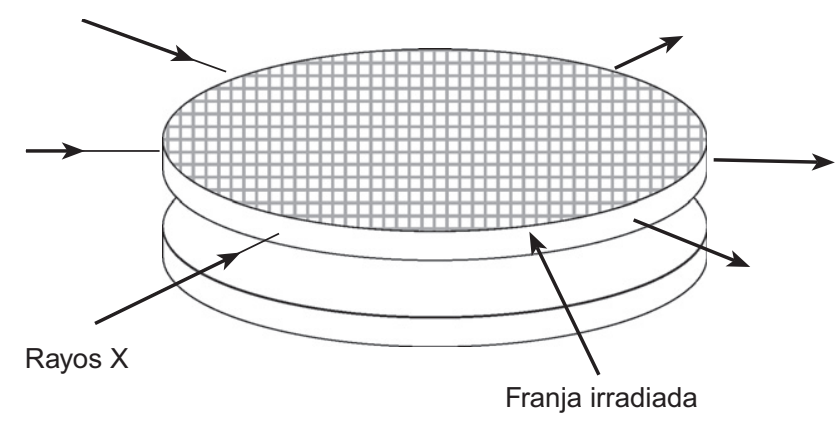

Figura 4 La trayectoria de los rayos $X$ está confinada al corte seleccionado debido a que los rayos pasan linealmente a través de los elementos contenidos en la malla.

\section{Mecánica pulmonar regional}

Como señalamos, la distribución del volumen insuflado depende esencialmente del gradiente vertical impuesto por la fuerza de gravedad sobre la superficie pleural y el parénquima pulmonar, y le confiere a este último la división funcional en R-ND y R-D. Según la posición que adopta el tórax (supina, lateral o prono), la $\mathrm{P}_{\mathrm{PL}}$ que rodea la superficie pulmonar dispuesta en la R-D es mayor que en la R-ND. Se ha descrito que por cada centímetro de altura, desde la R-ND a la R-D, esta presión incrementa en 0,2 a $0,5 \mathrm{~cm} \mathrm{H}_{2} \mathrm{O}^{31}$.

En VM controlada, la $\mathrm{P}_{\mathrm{PL}}$ se contrapone a la $\mathrm{P}_{\mathrm{ALV}}$. Como la $P_{P L}$ es menor en la R-ND, la $P_{T P}$ es mayor a este nivel. Es decir, frente a un mismo nivel de $P_{A L V}$, los alvéolos ubicados en la R-ND (anterior en posición supina) están sometidos a presiones de distensión más elevadas, poseen mayor radio y son más susceptibles a la sobredistensión que aquéllos ubicados en la R-D. Este fenómeno se podría atenuar limitando el $\mathrm{V}_{\mathrm{T}}$, aproximación que no garantiza el control total del colapso-reapertura alveolar en cada ciclo respiratorio $^{32,33}$.

Si mantenemos un $\mathrm{V}_{\mathrm{T}}$ constante e incrementamos la PEEP, desde el punto de vista teórico podemos enfrentar 2 situaciones: a) falta o escaso incremento del EELV (bajo o nulo PPR), y $b$ ) incremento del EELV (presencia de PPR). En el primer caso, el incremento de la PEEP sobredistenderá la población alveolar disponible y el $\mathrm{V}_{\mathrm{T}}$ puede superar la $\mathrm{P}_{\mathrm{TP}}$ crítica, y generar stress y strain $\left(\mathrm{V}_{\mathrm{T}}>\right.$ baby lung $)$. Sin embargo, la aplicación de PEEP aislado, sin efectuar una MRP previa, podría subestimar el verdadero PPR, ya que existen poblaciones alveolares con una presión crítica de apertura umbral (TOP, threshold opening pressures) mayor. En el segundo, el mismo $V_{\mathrm{T}}$ se distribuirá en un mayor número de unidades alveolares con la consiguiente reducción de la $\mathrm{P}_{\mathrm{TB}}$ limitando el strain $\left(\mathrm{V}_{\mathrm{T}}<\right.$ baby lung). Por lo tanto, la aplicación de una MRP previo al ajuste de la PEEP se sustenta en la presencia de poblaciones alveolares con diferentes $\mathrm{TOP}^{22,34}$. La primera afección se asocia con un parénquima pulmonar donde predominaría la inundación alveolar, y la segunda se asocia con un pulmón de esponja.

\section{Estrategias para ajustar la ventilación}

\section{A. Uso de $V_{T}$ bajo y presión positiva espiratoria final según fracción inspirada de oxígeno}

La estrategia ventilatoria propuesta por el estudio conducido por ARDSnet, preconiza minimizar el strain pulmonar mientras se mantiene el intercambio de gases mínimo aceptado ${ }^{3,35}$. Estas metas se logran usando $\mathrm{V}_{\mathrm{T}}$ de 4 a $6 \mathrm{ml} / \mathrm{kg}$ (peso corporal ideal) con fracción inspirada de oxígeno $\left(\mathrm{FiO}_{2}\right)$ y PEEP programadas, usando una tabla que combina niveles bajos de PEEP con altos niveles de $\mathrm{FiO}_{2}$, apuntando al menor PEEP para mantener una presión meseta inspiratoria $<30 \mathrm{cmH}_{2} \mathrm{O}$ y una oxigenación arterial de entre el 88 y el 95\%. Esta aproximación obtuvo una reducción de la mortalidad del $22 \%{ }^{3}$. Empero, esta estrategia se ha considerado dicotómica, ya que comparte la prevención de la distensión atribuida al $\mathrm{V}_{\mathrm{T}}$ bajo y limita la excursión alveolar en inspiración y, potencialmente, la apertura de unidades colapsadas, condicionando desreclutamiento pulmonar progresi$v^{36,37}$. El fenómeno de reclutamiento/desreclutamiento puede acontecer alrededor de 30.000 veces por día y la lesión consiguiente parece ser igual o incluso mayor que la provocada por sobredistensión ${ }^{20,34}$.

B. Curva presión-volumen inspiratoria

Para ajustar la PEEP individualmente se ha programado la VM según las características de la mecánica toracopulmonar obtenida a través de una curva presión-volumen (curva P-V) inspiratoria. En LPA/SDRA, esta curva es generalmente sigmoidal y describe 3 segmentos: inferior, zona de baja distensibilidad que corresponde a alvéolos colapsados que poseen un TOP similar; intermedio, donde la distensibilidad se mantiene constante durante la insuflación, y superior, de baja distensibilidad, vinculado con el fenómeno de sobredistensión. La intersección entre el segmento intermedio con el inferior y el superior determinan el punto de inflexión inferior (PII) y el punto de inflexión superior, respectivamente. El segmento intermedio corresponde a la distensibilidad lineal $^{34}$ (fig. 5).

Esta curva se puede obtener con técnicas estáticas o cuasiestáticas. Entre las estáticas destacan la superjeringa y la oclusión múltiple ${ }^{38,39}$. La técnica cuasiestática permite estudiar la mecánica toracopulmonar en clínica; sin embargo, la determinación de los puntos de inflexión posee gran variabilidad intraobservador e interobservador $^{40}$. Se ha propuesto el análisis en tiempo real usando ecuaciones polinomiales ${ }^{41,42}$.

Al ajustar la PEEP según el valor del $\mathrm{PII}+2 \mathrm{~cm} \mathrm{H}_{2} \mathrm{O}$ se ha logrado, además de optimizar el intercambio de gases ${ }^{43}$, reducir los niveles de mediadores inflamatorios ${ }^{44}$ y la mortalidad ${ }^{45,46}$; sin embargo, el uso del PII como indicador de reclutamiento posee limitaciones. La aplicación de PEEP y MRP puede aumentar el EELV a través de 2 mecanismos opuestos: uno, por incremento de la proporción de alvéolos aireados al final de la espiración (reclutamiento) o debido a la insuflación de regiones pulmonares previamente abiertas (sobredistensión) ${ }^{47}$. Existe evidencia consistente de la incorporación progresiva de nuevas unidades alveolares por encima del PII, y por tanto, la pendiente lineal no refleja sólo el incremento del radio alveolar de las unidades previamente abiertas (insuflación tipo globo [balloon-like]), sino también la apertura secuencial de nuevos alvéolos. Esto se ha demostrado tanto mediante estudios de mecánica pulmonar como con TC de tórax ${ }^{29,48,49}$. 


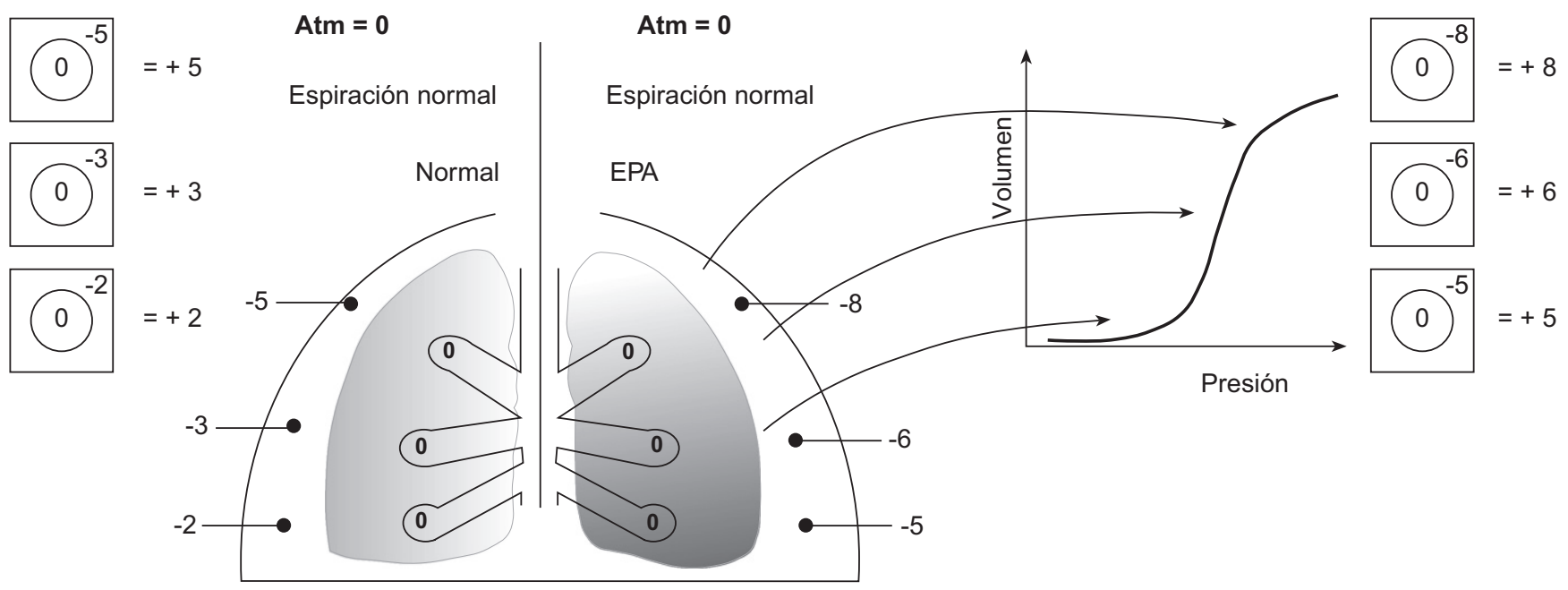

Figura 5 Curva presión-volumen, rama inspiratoria que representa la presión impuesta en el tejido pulmonar y los cambios gravitacionales de la presión pleural.

El grupo de Amato fue el que aportó la evidencia más consistente de que el reclutamiento alcanzado con el uso del PII es insuficiente; comparó la oxigenación y la presencia de tejido pulmonar no aireado en la TC de tórax bajo 2 estrategias: la primera, guiada por $\mathrm{PII}+2$ $\mathrm{CmH}_{2} \mathrm{O}$ y la segunda, con PEEP ajustado por un protocolo de titulación descendente de la PEEP (TD-PEEP), tras aplicar $\mathrm{MRP}^{50}$. Aquí se dilucidó que las presiones necesarias para sobreponerse al colapso en adultos con SDRA son mucho mayores que la predicha por la teoría de la presión sobreimpuesta, lo que indica la presencia de otros factores involucrados, tales como: incremento de la tensión superficial, aumento de la presión intraabdominal $u$ otros aún desconocidos ${ }^{51}$.

C. Titulación descendente de la presión positiva espiratoria final

La TD-PEEP después de haber efectuado una MRP se ha propuesto para disminuir la proporción de tejido pulmonar no aireado y limitar el fenómeno de reclutamiento/ desreclutamiento cíclico $22,51,52$. Esta técnica se fundamenta en la presencia de poblaciones alveolares con diferentes TOP y en la diferencia que existe entre las presiones necesarias para abrir el pulmón, de aquéllas requeridas para sostenerlo abierto (histéresis).

No es fácil determinar el escalón óptimo de PEEP, ya que la aparición de colapso durante la TD-PEEP debería comenzar con cambios localizados de la distensibilidad pulmonar, principalmente en la R-D, lo que corresponde a pérdida de unidades funcionales que la gravedad comprime. Estos cambios deberían preceder al deterioro de la distensibilidad pulmonar global y, eventualmente, al deterioro del intercambio de gases producto de la vasoconstricción hipóxica refleja ${ }^{53}$.

La distensibilidad de la R-D disminuye significativamente cuando el nivel de PEEP cae por debajo de $21 \mathrm{cmH}_{2} \mathrm{O}$, umbral asociado con disminución de la aireación en esta zona. El nivel óptimo de PEEP encontrado al utilizar el intercambio de gases y la distensibilidad dinámica fue de 20 y $16 \mathrm{cmH}_{2} \mathrm{O}$, respectivamente. Con el alivio de la sobredistensión secundario al descenso de la PEEP, la distensibilidad de la R-ND mejora progresivamente, lo que contrabalancea el deterioro de la distensibilidad en la R-D. En consecuencia, la distensibilidad pulmonar global alcanza su máximo con un promedio de PEEP menor al umbral de colapso real. Existe evidencia de que con el análisis cuantitativo de imágenes adquiridas con la tomografía de impedancia eléctrica (EIT, electrical impedance tomography) es posible detectar el colapso regional antes de que cambios en la distensibilidad pulmonar global y la gasometría lo evidencien ${ }^{53,54}$.

D. Curva presión-volumen espiratoria

La rama espiratoria de la curva P-V describe el vaciamiento pulmonar, que se inicia una vez que la insuflación pulmonar ha alcanzado la capacidad pulmonar total. Para estudiar esta porción se ha utilizado la técnica de superjeringa y maniobras de descenso paso a paso de presión positiva continua en la vía aérea, con el fin de obtener la presión meseta (flujo cero o mínimo) en cada escalón de volumen durante la espiración ${ }^{55,56}$.

La PEEP es una maniobra espiratoria, por lo que el nivel óptimo debería estar correlacionado con el desreclutamiento. Albaiceta demostró que la pérdida de aireación y el desreclutamiento fueron significativos sólo con presiones inferiores al punto de máxima curvatura de la rama espiratoria de la curva P-V. Además, en este punto se observó la mayor cantidad de tejido normalmente aireado, medido con TC de tórax, comparado con imágenes obtenidas a la altura del PII. Un aspecto destacable fue que el tejido hiperinsuflado no incrementó significativamente ${ }^{56}$.

E. Tomografía de impedancia eléctrica

Los iones disueltos en los tejidos corporales permiten la conducción eléctrica, y por tanto, a mayor contenido mejor conducción; por ejemplo, el hueso y el pulmón insuflado son pobres conductores, en cambio, el músculo y el pulmón colapsado son buenos conductores.

La conductividad se expresa en siemens por metro $(\mathrm{S} / \mathrm{m})$ y corresponde a la conductabilidad de una unidad cúbica de tejido. Su recíproco, la impedancia, es la capacidad que tiene una unidad cúbica de un determinado tejido 


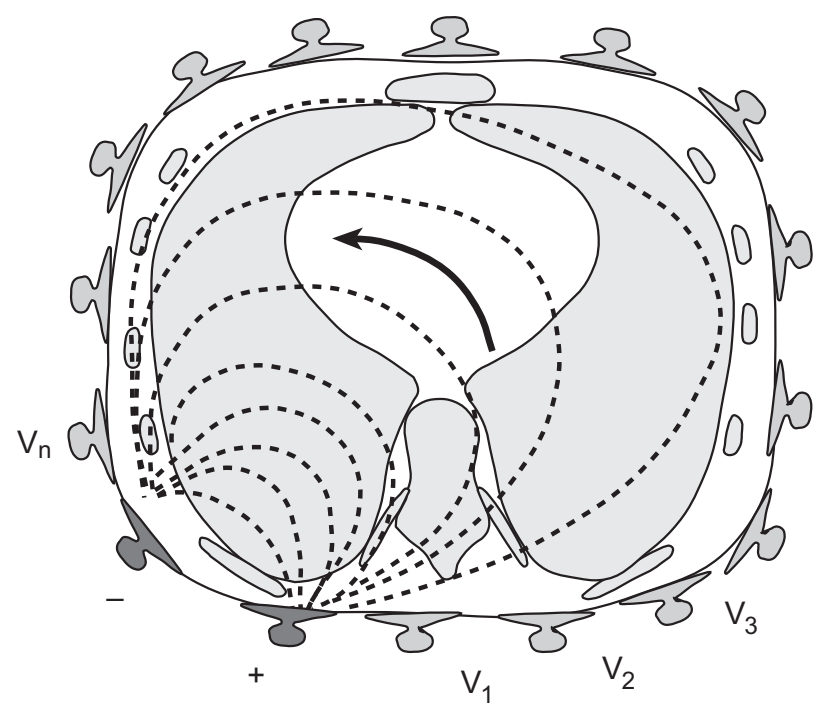

Figura 6 Distribución de la corriente eléctrica emitida por los electrodos activos. La recepción se produce en los electrodos pasivos ubicados al final del recorrido (flecha), proceso que se repite $(50 \mathrm{c} / \mathrm{s})$.

para oponerse a la conducción eléctrica. Sobre la base de estos principios, la EIT es capaz de generar imágenes a partir de la distribución de los valores absolutos de impedancia de los diferentes tejidos corporales, y en el caso de órganos dinámicos, como el pulmón, es posible estimar las variaciones de volumen gracias a los cambios de impedancia producidos por el ingreso y la salida de aire $^{57,58}$.

Las imágenes pulmonares se obtienen con la inyección repetida y alternada de corriente de pequeña intensidad $(\sim 5 \mathrm{~mA})$ en sentido rotatorio a través de electrodos de superficie (12 a 32) aplicados en un plano alrededor del tórax $\left(5 .^{\circ}\right.$ espacio intercostal). El método es capaz de medir las diferencias de potencial entre pares de electrodos adyacentes conductores y pares de electrodos pasivos. Los datos de voltaje se analizan para producir imágenes, proceso denominado reconstrucción ${ }^{57}$ (fig. 6). La capacidad que posee esta técnica para obtener imágenes a partir de las variaciones de impedancia ocurridas durante un proceso fisiológico, la pone en ventaja frente a los métodos que describen la anatomía, como la TC y la resonancia magnética. Además, si se considera que la distribución de la corriente a través de los tejidos no es lineal, es posible obtener imágenes pulmonares más representativas del tórax $(10-15 \mathrm{~cm}$ de espesor) ${ }^{23}$.

Las modificaciones en la aireación pulmonar se acompañan de cambios correspondientes de la impedancia regional, que puede cuantificarse y graficarse matemáticamente. Esto último es posible gracias a la excelente resolución temporal que posee actualmente la EIT $(50 \mathrm{c} / \mathrm{s})$, lo que permite adquirir muestras con alta frecuencia de las características del llenado y vaciamiento pulmonar ${ }^{23,57}$ (fig. 7).

Esta técnica permite evaluar diversos aspectos de la función pulmonar, tales como la distribución de la aireación pulmonar regional con diferentes condiciones ventilatorias; destaca la relación entre R-ND y R-D

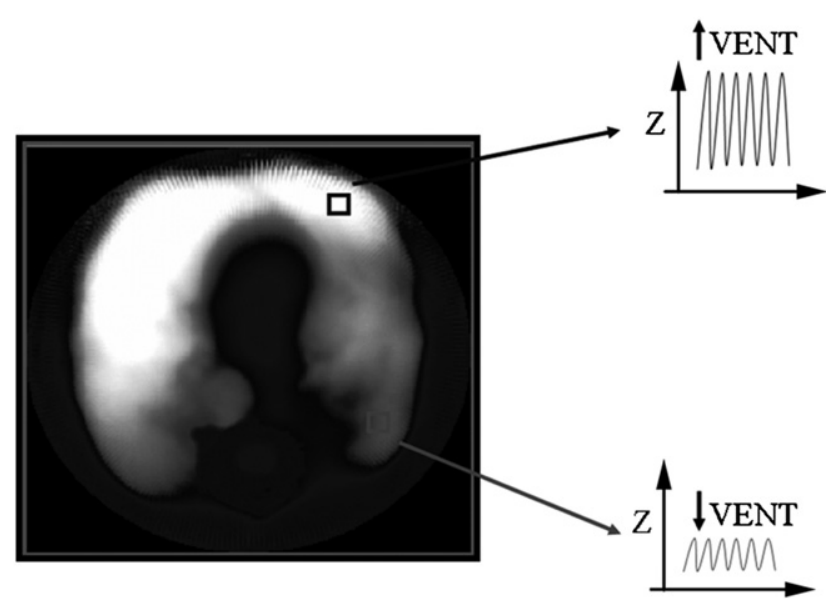

Figura 7 Imagen obtenida con electrical impedance tomography. Gráfico superior (upper): cambios importantes de la señal de impedancia; gráfico inferior (lower): cambios mínimos de esta señal.

(coeficiente upper/lower), especialmente luego de cambios del $V_{T}$ y la PEEP (fig. 8). La EIT ha mostrado muy buena correlación con diversos métodos de referencia, tales como: espirometría ${ }^{58}$, TC de tórax ${ }^{59}$ y TC por emisión de electrones ${ }^{60}$.

En resumen, por su capacidad para cuantificar dinámicamente la distribución esternodorsal de la ventilación, sin interrumpir la ventilación ni exponer al paciente a los efectos nocivos de la radiación, este método se proyecta como la herramienta de monitorización ideal para identificar la estrategia ventilatoria que asegure una distribución más homogénea de la ventilación.

F. Reclutamiento anatómico versus reclutamiento funcional

Actualmente, existe discusión acerca de 2 tipos de reclutamiento: "anatómico" y "funcional". El primero se refiere a la reducción de la masa de tejido pulmonar colapsado medido por TC de tórax, y el segundo se refiere a la disminución del cortocircuito intrapulmonar estimado a partir del contenido arterial y venoso mixto de oxígeno. Se ha indicado que el desplazamiento de la perfusión puede actuar como mecanismo causante de la disociación entre la ganancia de aireación inducida por la PEEP y la contribución que debería tener la región reexpandida en la fracción global del cortocircuito intrapulmonar.

En caso de reclutamiento parcial, el desplazamiento de la perfusión hacia las regiones dependientes (colapsadas) inducido por la PEEP puede prevalecer sobre el efecto benéfico esperado al mejorar la aireación sobre el cortocircuito regional. En contraste, al emplear una MRP máxima seguida de un nivel de PEEP óptimo, la reexpansión extensa del tejido pulmonar colapsado atenuaría esta disociación. Es así como las divergencias entre reclutamiento "anatómico" y "funcional" podrían depender de la estrategia seleccionada para titular la PEEP ${ }^{35,61-63}$. Por otra parte, los efectos de las MRP pueden verse opacados por su eventual impacto sobre el gasto cardíaco y transporte de oxígeno; es decir: 
$\mathrm{FIO}_{2}=100 \% ; \mathrm{PEEP}=25 \mathrm{cmH}_{2} \mathrm{O} ; \mathrm{V}_{\mathrm{T}}=4 \mathrm{~mL} / \mathrm{Kg}$

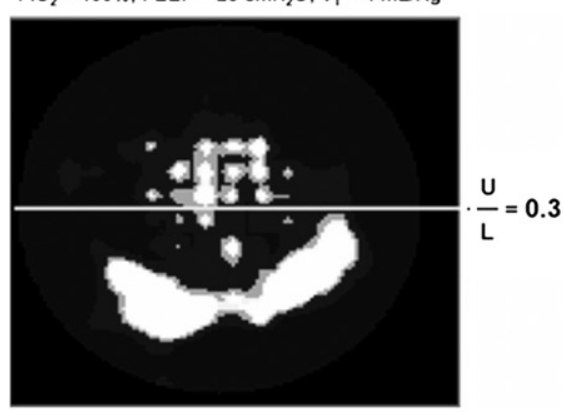

$\mathrm{FIO}_{2}=100 \% ;$ PEEP $=15 \mathrm{cmH}_{2} \mathrm{O} ; \mathrm{V}_{\mathrm{T}}=4 \mathrm{~mL} / \mathrm{Kg}$
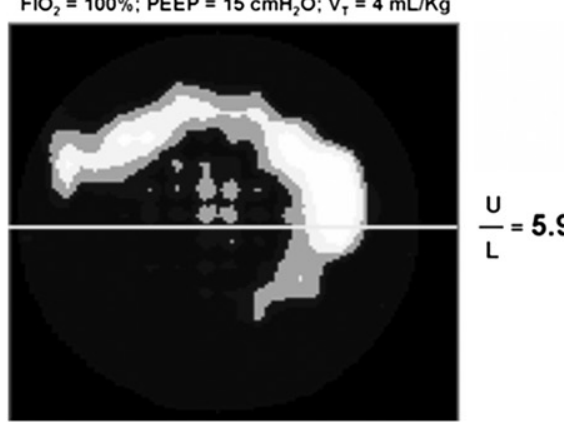

$\mathrm{FIO}_{2}=100 \% ; \mathrm{PEEP}=21 \mathrm{cmH}_{2} \mathrm{O} ; \mathrm{V}_{\mathrm{T}}=4 \mathrm{~mL} / \mathrm{Kg}$

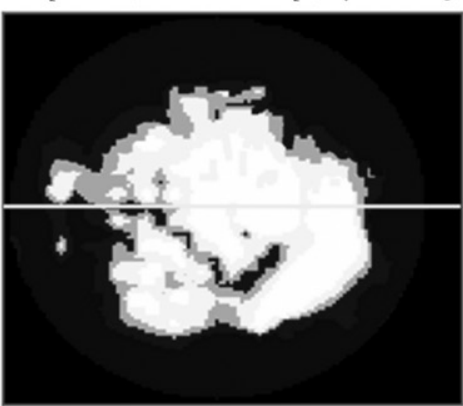

$\mathrm{FIO}_{2}=100 \% ; \mathrm{PEEP}=13 \mathrm{cmH}_{2} \mathrm{O} ; \mathrm{V}_{\mathrm{T}}=4 \mathrm{~mL} / \mathrm{Kg}$

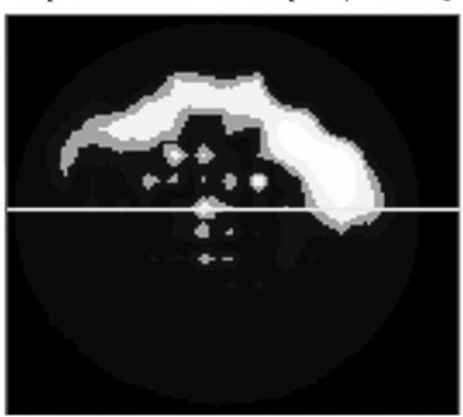

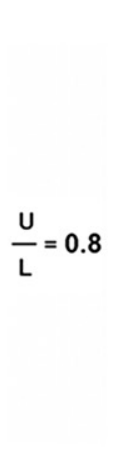

$\frac{U}{L}=6.7$
$\mathrm{FIO}_{2}=100 \% ; \mathrm{PEEP}=17 \mathrm{cmH}_{2} \mathrm{O} ; \mathrm{V}_{\mathrm{T}}=4 \mathrm{~mL} / \mathrm{Kg}$

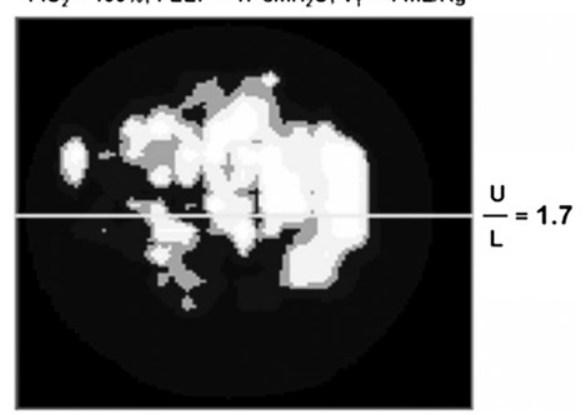

$\mathrm{FIO}_{2}=100 \% ; \mathrm{PEEP}=0 \mathrm{cmH}_{2} \mathrm{O} ; \mathrm{V}_{\mathrm{T}}=4 \mathrm{~mL} / \mathrm{Kg}$

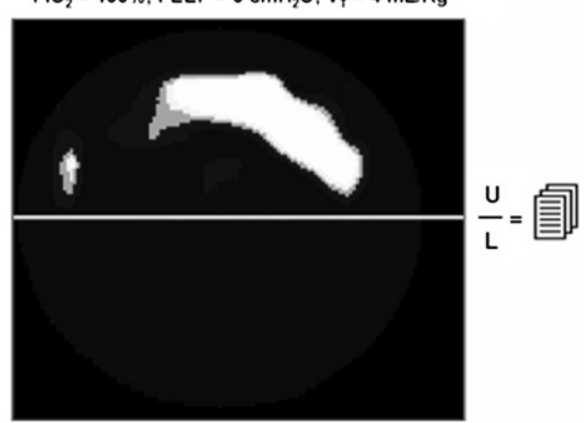

Figura 8 Relación upper/lower con diferentes niveles de presión positiva espiratoria final (PEEP). Nótese el bajo nivel de impedancia en la mitad superior (upper) con (PEEP) de $25 \mathrm{cmH}_{2} \mathrm{O}$. La homogenización de la impedancia con PEEP de $21 \mathrm{cmH} \mathrm{H}_{2} \mathrm{O}$ (coeficiente upper/lower de 0,8 ) y el colapso progresivo mostrado por la caída de la impedancia en las regiones inferiores (lower) con PEEP de 17, 15, 13 y $0 \mathrm{cmH}_{2} \mathrm{O}$. Con permiso del Dr. Marcelo B. P. Amato, Universidad de Sao Paulo, Brasil. FiO 2 : fracción inspirada de oxígeno.

una correcta interpretación del beneficio de la MRP requiere una evaluación simultánea del status hemodinámico y del cortocircuito intrapulmonar. El efecto hemodinámico de la realización de curvas P/V y MRP ha sido inconsistente ${ }^{64,65}$.

\section{Discusión}

El SDRA es una enfermedad grave cuya incidencia y mortalidad representan un problema de salud pública. Desde el punto de vista fisiopatológico se define por una alteración de la membrana alveolocapilar producto de un estímulo proinflamatorio local o sistémico que se traduce en grados variables de edema intersticial, ocupación alveolar y colapso de los espacios aéreos.

Existen diversas técnicas para evaluar las características mecánicas, estructurales y funcionales del tejido pulmonar en estos pacientes. A pesar de esto, la programación de la VM sigue siendo motivo de controversia.

Hoy sabemos que los beneficios de utilizar $V_{T}$ de entre 6 y $8 \mathrm{ml} / \mathrm{kg}$ de peso ideal se obtienen siempre y cuando la presión meseta no exceda los $30 \mathrm{cmH}_{2} \mathrm{O}^{32}$. El uso de PEEP elevado, en cambio, no ha demostrado ventajas consistentes, debido muy probablemente a la heterogeneidad de las poblaciones estudiadas ${ }^{66}$.

En trabajos más recientes, metas secundarias, tales como: días libres de VM, número de disfunciones orgánicas, presencia de hipoxemia refractaria y necesidad de tratamientos de rescate, mostraron una tendencia favorable en pacientes asignados, sin distinción, a niveles elevados de
PEEP ${ }^{67,68}$. Aunque el impacto sobre la mortalidad fue modesto, la PEEP alta puede beneficiar al subgrupo con relación $\mathrm{PaO}_{2} / \mathrm{FiO}_{2}$ baja ${ }^{36}$. Estudios futuros en pacientes con un compartimento no aireado extenso que utilicen un nivel de PEEP ajustado por respuesta fisiológica post-MRP deberían dilucidar el rol verdadero de esta maniobra en el tratamiento del SDRA grave.

$\mathrm{Si}$ asumimos que homogeneizar la distribución del $\mathrm{V}_{\mathrm{T}}$ en las R-ND y R-D es fundamental, no sólo para optimizar el intercambio de gases, sino también para minimizar la VILI y atenuar el DOM, la exploración del PPR y el ajuste descendente de la PEEP podrían convertirse en una estrategia habitual en pacientes con SDRA grave, a la vez que se asegure estabilidad hemodinámica.

En la práctica, la concepción actual de "VM protectora" implica efectuar un ajuste individualizado de la PEEP y el $V_{T}$. No obstante, si se integra toda la evidencia disponible, la búsqueda de la PEEP "ideal" y de la estrategia protectora óptima son aún tareas pendientes.

\section{Conflicto de intereses}

Los autores declaran no tener ningún conflicto de intereses.

\section{Agradecimientos}

Al profesor Harki Tanaka de la Facultad de Medicina de la Universidad de Sao Paulo, Brasil, por la generosidad con que nos ha entregado sus conocimientos. 


\section{Bibliografía}

1. Ashbaugh DG, Bigelow DB, Petty TL, Levine BE. Acute respiratory distress in adults. Lancet. 1967;2:319-23.

2. Amato MB, Barbas CS, Medeiros DM, Magaldi RB, Schettino GP, Lorenzi-Filho $\mathrm{G}$, et al. Effect of a protective-ventilation strategy on mortality in the acute respiratory distress syndrome. N Engl J Med. 1998;338:347-54.

3. ARDSNet. Ventilation with lower tidal volumes as compared with traditional tidal volumes for acute lung injury and the acute respiratory distress syndrome. $N$ Engl J Med 2000;342:1301-8.

4. Phua J, Badia JR, Adhikari NK, Friedrich JO, Fowler RA, Singh $J M$, et al. Has mortality from acute respiratory distress syndrome decreased over time? A systematic review Am J Respir Crit Care Med. 2009;179:220-7.

5. Borges JB, Carvalho CR, Amato MB. Ventilation strategies for acute lung injury and acute respiratory distress syndrome. JAMA. 2008;300:41; author reply 41-2.

6. Borges JB, Carvalho CR, Amato MB. Lung recruitment in patients with ARDS. N Engl J Med. 2006;355:319-20; author reply 321-2.

7. Rubenfeld GD, Caldwell E, Peabody E, Weaver J, Martin DP, Neff $M$, et al. Incidence and outcomes of acute lung injury. $N$ Engl J Med. 2005;353:1685-93.

8. Frutos-Vivar F, Nin N, Esteban A. Epidemiology of acute lung injury and acute respiratory distress syndrome. Curr Opin Crit Care. 2004;10:1-6.

9. Ware LB, Matthay AM. The acute respiratory distress syndrome. N Engl J Med. 2000;242:1334-49.

10. Pelosi P, D’Andrea L, Vitale G, Pesenti A, Gattinoni L. Vertical gradient of regional lung inflation in adult respiratory distress syndrome. Am J Respir Crit Care Med. 1994;149:8-13.

11. Hubmayr R. Perspective on lung injury and recruitment: A sceptical look at the opening and collapse story. Am J Respir Crit Care Med. 2002;165:1647-53.

12. Schuster DP, Stark T, Stephenson J, Royal H. Detecting lung injury in patients with pulmonary edema. Intensive Care Med. 2002;28:1246-53.

13. Dos Santos CC, Slutsky S. Cellular responses to mechanical stress invited review: Mechanisms of ventilator-induced lung injury: A perspective. J Appl Physiol. 2000;89:1645-55.

14. Dreyfuss D, Soler P, Basset G, Saumon G. High inflation pressure pulmonary edema: Respective effects of high airway pressure, high tidal volume and positive end-expiratory pressure. Am Rev Respir Dis. 1988;137:1159-64.

15. Dreyfuss D, Saumon G. Ventilator-induced lung injury: Lesson from experimental studies. Am J Respir Crit Care Med. 1998;157:294-323.

16. Webb HH, Tierney DF. Experimental pulmonary edema due to intermittent positive pressure ventilation with high inflation pressure: Protection by positive end-expiratory pressure. Am Rev Respir Dis. 1974;110:556-65.

17. Slutsky AS, Tremblay LN. Multiple system organ failure. Is mechanical ventilation a contributing factor? Am J Respir Crit Care Med. 1998;157:1721-5.

18. Mead J, Takishima T, Leith D. Stress distribution in lungs: A model of pulmonary elasticity. J Appl Physiol. 1970;28:596-608.

19. Rouby JJ. Lung overinflation: The hidden face of alveolar recruitment. Anesthesiology. 2003;99:2-4.

20. Muscedere JG, Mullen JBM, Gan K, Slutsky AS. Tidal ventilation at low airway pressure can augment lung injury. Am J Respir Crit Care Med. 1994;149:1327-34.

21. Halter JM, Steinberg JM, Schiller HJ, Da Silva M, Gatto LA, Landas $S$, et al. Positive end-expiratory pressure after a recruitment maneuver prevents both alveolar collapse and recruitment/derecruitment. Am J Respir Crit Care Med. 2003;167:1620-6.
22. Borges JB, Okamoto VN, Matos GF, Caramez MP, Arantes PR, Barros F, et al. Reversibility of lung collapse and hypoxemia in early acute respiratory distress syndrome. Am J Respir Crit Care Med. 2006;174:268-78.

23. Okamoto VN, Borges JB, Amato MB. Recruitment maneuvers in ARDS. En: Slutsky AS, Brochard L, editores. Update in intensive care and emergency medicine: Mechanical ventilation. Berlin; Heidelberg; New York: Springer-Verlag; 2004. p. 335-52.

24. Suárez Sipmann F. Utilidad de las maniobras de reclutamiento (PRO). Med Intensiva. 2009;33:134-9.

25. Ochagavia A, Blanch L, López-Aguilar J. Utilidad de las maniobras de reclutamiento (contra). Med Intensiva. 2009;33:139-43.

26. Gattinoni L, Pesenti A. The concept of "baby lung". Intensive Care Med. 2005;31:776-84.

27. Marini JJ, Gattinoni L. Ventilatory management of acute respiratory distress syndrome: A consensus of two. Crit Care Med. 2004;22:250-5.

28. Chiumello D, Carlesso E, Cadringher P, Caironi P, Valenza1 F, Polli $F$, et al. Lung stress and strain during mechanical ventilation for acute respiratory distress syndrome. Am J Respir Crit Care Med. 2008;178:346-55.

29. Gattinoni L, Caironi P, Pelosi P, Goodman LR. What has computed tomography taught us about the acute respiratory distress syndrome? Am J Respir Crit Care Med. 2001;164:170111.

30. Hayhurst MD, Macnee W, Flenley DC, Wright D, Mclean A, Lamb $D$, et al. Diagnosis of pulmonary emphysema by computerised tomography. Lancet. 1984;11:320-2.

31. Lai-Fook SJ, Rodarte JR. Pleural pressure distribution and its relationship to lung volume and interstitial pressure. J Appl Physiol. 1991;70:967-8.

32. Eichacker PQ, Gerstenberger EP, Banks SM, Cui X, Natanson C. Meta-analysis of acute lung injury and acute respiratory distress syndrome trials testing low tidal volumes. Am J Respir Crit Care Med. 2002;166:1510-4.

33. Terragni PP, Rosboch G, Tealdi A, Corno E, Menaldo E, Davini O, et al. Tidal hyperinflation during low tidal volume ventilation in acute respiratory distress syndrome. Am J Respir Crit Care Med. 2007;175:160-6.

34. Marini JJ, Amato MB. Lung recruitment during ARDS. En: Vincent $\mathrm{JL}$, editor. Update in intensive care and emergency medicine. Berlin; Heidelberg; New York: Springer-Verlag; 1998. p. 236-57.

35. Brower RG, Lanken PN, Maclntyre N, Matthay MA, Morris A, Ancukiewicz $M$, et al. Higher versus lower positive endexpiratory pressures in patients with the acute respiratory distress syndrome. N Engl J Med. 2004;351:327-36.

36. Gordo-Vial F, Gómez-Tello V, Palencia-Herrejón E, Latour-Pérez J. Impact of two new studies on the results of a meta-analysis on the application of high PEEP in patients with acute respiratory distress sindrome. Med Intensiva. 2008;32:316-7.

37. Cereda M, Foti G, Musch G, Sparacino ME, Pesenti A. Positive end- expiratory pressure prevent the loss of respiratory compliance during low tidal volume ventilation in acute lung injury patient. Chest. 1996;109:480-5.

38. Lee WL, Stewart TE, Macdonald R, Lapinsky S, Banayan D, Hallett $D$, et al. Safety of pressure-volume curve measurement in acute lung injury and ARDS using a syringe technique. Chest. 2002;121:1595-601.

39. Suratt PM, Owens DH. A pulse method of measuring respiratory system compliance in ventilated patients. Chest. 1981;80:34-8.

40. Dall'Ava-Santucci J, Armaganidis A, Brunet F, Dhainaut JF, Chelucci GL, Monsallier JF, et al. Causes of error of respiratory pressure-volume curves in paralyzed subjects. J Appl Physiol. 1988;64:42-9.

41. Harris RS, Hess DR, Venegas JG. An objective analysis of the pressure-volume curve in the acute respiratory distress syndrome. Am J Respir Crit Care Med. 2000;161:432-9. 
42. Tomicic V, Fidanza L, Espinoza M, Graf J, Canals C. Use of a sigmoidal equation to analyze the pressure volume curve obtained by the low flow method. Critical Care. 2005;9:S44.

43. Tomicic V, Molina J, Graf J, Espinoza M, Antúnez M, Errazuriz I, et al. Ajuste de la ventilación mecánica guiado por curva presión-volumen de flujo lento en pacientes con síndrome de distress respiratorio agudo e injuria pulmonar aguda. Rev Med Chil. 2007; 135:307-17.

44. Ranieri VM, Suter PM, Tortorella C, De Tullio R, Dayer JM, Brienza $A$, et al. Effect of mechanical ventilation on inflammatory mediators in patients with acute respiratory distress syndrome: A randomized controlled trial. JAMA. 1999;282:54-61.

45. Amato MBP, Barbas CSV, Medeiros DM, Magaldi RB, Schettino GP, Lorenzi-Filho $\mathrm{G}$, et al. Effect of a protective-ventilation strategy on mortality in the acute respiratory distress syndrome. N Engl J Med. 1998;338:347-54.

46. Villar J, Kacmarek R, Pérez-Méndez L, Aguirre-Jaime A. For the ARIES Network. A high positive end-expiratory pressure, low tidal volume ventilatory strategy improves outcome in persistent acute respiratory distress syndrome: A randomized, controlled trial. Crit Care Med. 2006;34:1311-8.

47. Grasso S, Fanelli V, Cafarelli A, Anaclerio R, Amabile M, Ancona $G$, et al. Effects of high versus low positive end-expiratory pressure in acute respiratory distress syndrome. Am J Respir Crit Care Med. 2005;171:1002-8.

48. Jonson B, Richard JC, Straus C, Mancebo J, Lemaire F, Brochard L. Pressure-volume curves and compliance in acute lung injury. Evidence of recruitment above the lower inflection point. Am J Respir Crit Care Med. 1999;159:1172-8.

49. Crotti S, Mascheroni D, Caironi P, Pelosi P, Ronzoni G, Mondino $M$, et al. Recruitment and derecruitment during acute respiratory failure. A clinical study. Am J Respir Crit Care Med. 2001;164:131-40.

50. Borges JB, Caramez MPR, Gaudencio AMAS. Lung recruitment at airway pressures beyond $40 \mathrm{cmH}_{2} \mathrm{O}$ : Physiology, mechanics and spiral CT analysis. Am J Respir Crit Care Med. 2000;161:A48 (abstract).

51. Marini JJ, Amato MB. Lung recruitment during ARDS. Minerva Anestesiol. 2000;66:314-9.

52. Hickling KG. Best compliance during a decremental, but not incremental, positive end-expiratory pressure trial is related to open lung positive end-expiratory pressure: A mathematical model of acute respiratory distress syndrome lungs. Am J Respir Crit Care Med. 2000;66:314-9.

53. Borges JB, Costa ELV, Beraldo MA, Gomes S, Volpe MS, Carvalho CRR, et al. A bedside and real-time monitor to detect airspace collapse in patients with ALI/ARDS [abstract]. Am J Respir Crit Care Med. 2006:3:A377.

54. Beraldo MA, Reske A, Borges JB, Costa ELV, Gomes S, Volpe MS, et al. PEEP titration by EIT (electric impedance tomography): Correlation with multislice CT [abstract]. Am J Respir Crit Care Med. 2006:3:A64.

55. Albaiceta GM, Piacentini E, Villagrá A, López-Aguilar J, Taboada F, Blanch L. Application of continuous positive airway pressure to trace static pressure-volume curves of the respiratory system. Crit Care Med. 2003;31:2514-9.
56. Albaiceta GM, Taboada F, Parra D, Luyando LH, Calvo J, Menéndez $\mathrm{R}$, et al. Tomographic study of the inflection points of the pressure-volume curve in acute lung injury. Am J Respir Crit Care Med. 2004;170:1066-72.

57. Putensen C, Zinserling J, Wrigge H. Electrical impedance tomography for monitoring of regional ventilation in critical ill patients. En: Vincent JL, editor. Yearbook of intensive care and emergency medicine. Berlin; Heidelberg; New York: SpringerVerlag; 2006. p. 448-57.

58. Hahn G, Sipinkova I, Baisch F, Hellige G. Changes in the thoracic impedance distribution under different ventilatory conditions. Physiol Meas. 1995;16:A161-73.

59. Victorino JA, Borges JB, Okamoto VN, Caramez MP, Arantes PR, Barros $F$, et al. Imbalances in regional lung ventilation: $A$ validation study on electrical impedance tomography. Am J Respir Crit Care Med. 2004;169:791-800.

60. Frerichs I, Hinz J, Herrmann P, Weisser G, Hahn G, Dudykevych $\mathrm{T}$, et al. Detection of local lung air content by electrical impedance tomography compared with electron beam CT. J Appl Physiol. 2002;93:660-6.

61. Musch G, Bellani G, Vidal Melo MF, Harris RS, Winkler T, Schroeder T, et al. Relation between shunt, aeration, and perfusion in experimental acute lung injury. Am J Respir Crit Care Med. 2008;177:292-300.

62. Cressoni M, Caironi P, Polli F, Carlesso E, Chiumello D, Cadringher P, et al. Anatomical and functional intrapulmonary shunt in acute respiratory distress syndrome. Crit Care Med. 2008;36:669-75.

63. Suárez-Sipmann F, Bohm SH, Tusman G, Pesch T, Thamm O, Reissmann $\mathrm{H}$, et al. Use of dynamic compliance for open lung positive end-expiratory pressure titration in an experimental study. Crit Care Med. 2007;35:214-21.

64. Pestaña D, Royo C, Hernández-Gancedo C, Martínez-Casanova E, Criado A. Hemodynamic variability caused by pressurevolume plotting and alveolar recruitment maneuvers in patients with adult respiratory distress syndrome. Rev Esp Anestesiol Reanim. 2008;55:348-54.

65. Carvalho CRR, Barbas CSV, Medeiros DM, Magaldi RB, Filho GL, Kairalla RA, et al. Temporal hemodynamic effects of permissive hypercapnia associated with ideal PEEP in ARDS. Am J Respir Crit Care Med. 1997; 156:1458-66.

66. The National Heart, Lung, and Blood Institute ARDS Clinical Trials Network. Higher versus lower positive end-expiratory pressures in patients with the acute respiratory distress syndrome N Engl J Med. 2004;351:327-36.

67. Mercat A, Richard JC, Vielle B, Jaber S, Osman D, Diehl, et al; Expiratory Pressure (Express) study group. Positive end-expiratory pressure setting in adults with acute lung injury and acute respiratory distress syndrome: A randomized controlled trial. JAMA. 2008;299:646-55.

68. Meade MO, Cook DJ, Guyatt GH, Slutsky AS, Arabi YM, Cooper DJ, et al. Lung Open Ventilation study investigators. Ventilation strategy using low tidal volumes, recruitment maneuvers, and high positive end-expiratory pressure for acute lung injury and acute respiratory distress syndrome: A randomized controlled trial. JAMA. 2008;299:637-45. 